\title{
Electronic structure modulation of graphene edges by chemical functionalization
}

\author{
Remi Taira, Ayaka Yamanaka*, and Susumu Okada ${ }^{\dagger}$ \\ Graduate School of Pure and Applied Sciences, University of Tsukuba, 1-1-1 Tennodai, Tsukuba, Ibaraki \\ 305-8571, Japan
}

\begin{abstract}
Using the density functional theory with the effective screening medium method, we study the electronic properties of graphene nanoribbons with zigzag edges which are terminated by hydrogen and functional groups of ketone, hydroxy, carbonyl, and carboxyl groups. Our calculations showed that the work function and electronic structures of the edges of the nanoribbons are sensitive to the functional groups attached to the edges. The nearly free electron state emerges in the vacuum region outside the hydroxylated edges and crosses the Fermi level, indicating the possibility of the negative electron affinity at the edges.
\end{abstract}

Graphene has attracted much attention in the fields of nanoscience and nanotechnology because of its unique electronic and geometric properties. ${ }^{1-5)}$ A honeycomb network of $\mathrm{sp}^{2} \mathrm{C}$ atoms with single-atom thickness causes a pair of linear dispersion bands at the Fermi level, resulting in remarkable carrier mobility of a few hundred thousand $\mathrm{cm}^{2} / \mathrm{Vs}$ which make graphene being emerging materials for high-speed switching devices in the next-generation semiconductor technology. In addition to the technological views, the linear dispersion bands lead to the unusual quantum Hall effect, making graphene a unique material also in fundamental sciences. These remarkable electronic properties of graphene have been elucidated to be fragile against the external conditions such as structural imperfections, structural corrugations, atom/molecule adsorptions, interactions with other graphene or substrates, and external electric field. ${ }^{6-11)}$ For example, graphene has an energy gap of a few tens of meV, adsorbed on insulating substrates depending on the substrate species. Atomic defects, edges, and interfaces with the other two-dimensional materials also cause peculiar edge/interface localized states depending on their edge/interface morphologies. ${ }^{12-15)}$ These facts imply that the physical properties and functionality of graphene can be tunable by controlling the hybrid structures with other foreign materials and external conditions.

\footnotetext{
${ }^{*}$ E-mail address: ayamanaka@comas.frsc.tsukuba.ac.jp

${ }^{\dagger}$ E-mail address: sokada@comas.frsc.tsukuba.ac.jp
} 
Edge functionalization is one of possible procedures to tailor the geometries, stability, and electronic properties of graphene nanoflakes. Since electronic properties of the functional groups are sensitive to their constituent elements and molecular conformations, the functionalization affects the stability and physical properties of graphene flakes. Indeed, the functionalization of graphene has been applied to synthesize nanoscale graphene from bottom-up and top-down procedures. ${ }^{16-20)}$ Although, the experiments have reported the synthesis of various functionalized graphene flakes, local and detailed electronic properties of functionalized graphene flakes are still unclear. Thus, in the present work, we aim to give theoretical insight into the fundamental properties of graphene nanoribbons whose edges are functionalized by various functional groups, unraveling their electrostatic and electronic properties. We consider the asymmetrically functionalized graphene nanoribbons with zigzag edges to elucidate the relative modulation of the work function to the hydrogenated edges by attaching ketone, hydroxyl, carbonyl, and carboxyl groups. Our calculations show that the work function and electronic structure of graphene edges are sensitive to the functional group species. The work function of the zigzag edge functionalized by hydroxyl groups is smaller than that of the hydrogenated edges. We also found that the nearly free electron (NFE) state substantially shifts downward and crosses the Fermi level, indicating the possibility of the negative electron affinity at the hydroxylated zigzag edge of graphene.

In this work, we studied the geometric and electronic structures of graphene nanoribbons with asymmetrically functionalized zigzag edges using density functional theory $(\mathrm{DFT})^{21,22)}$ implemented into the Simulation Tool for Atom TEchnology (STATE). ${ }^{23)}$ We used the local density approximation with the Perdew-Zunger functional form fitted to the Quantum Monte Carlo results for a homogeneous electron gas to calculate the exchange correlation potentials among interacting electrons. ${ }^{24,25)}$ Ultrasoft pseudopotentials generated by the Vanderbilt scheme were used to describe electron-ion interactions. ${ }^{26)}$ Valence wave functions and charge densities were expanded in terms of the plane wave basis set with cutoff energies of 25 and 225 Ry, respectively. Since the nanoribbons are asymmetrically functionalized by the hydrogen atoms and functional groups leading to the polarization between two edges, we adopted the effective screening medium (ESM) method ${ }^{27)}$ to avoid the unphysical dipole interaction with the periodic images within the frame work of the DFT with the plane wave basis set. Here, we set the effective screening mediums alongside edges of the nanoribbons with the relative permittivity of 1 , which mimic an open boundary condition at the two edges. 
The atomic structures were fully optimized until the force acting on each atom was less than $5 \mathrm{mRy} / \AA$ under the fixed lattice parameter along the ribbon of $0.49 \mathrm{~nm}$ which correspond with the double periodicity of that of the zigzag nanoribbons. Integration over the one-dimensional Brillouin zone was carried out using equidistant $k$-point sampling in which $4 k$-points were taken along ribbon direction, which correspond to the 8 $k$-point sampling for the primitive cell of graphene, which gives sufficient convergence for both geometric and electronic structures.

In the present work, we consider zigzag graphene nanoribbons with the ribbon width of $1.56 \mathrm{~nm}$ whose edge atomic sites are asymmetrically terminated by $\mathrm{H}$ atoms and functional groups. To clarify how the work function and electronic properties depend on the edge functional groups, we consider ketone, hydroxyl, carbonyl, and carboxyl groups as the representative functional groups (Fig. 1). For the carbonyl and carboxyl groups, to avoid the steric hindrance between adjacent edge atomic sites, the edge $\mathrm{C}$ sites are alternately terminated by these functional groups and hydrogen atoms. To simulate an isolated graphene nanoribbons, each graphene nanoribbon is separated by its periodic images by 6 and $10 \AA$ vacuum regions normal and lateral direction of the nanoribbon, respectively.

Figure 2 shows plane-averaged electrostatic potential of asymmetrically functionalized graphene nanoribbons as a function of the lateral direction of the ribbons. Because of the chemical difference between $\mathrm{H}$ atoms and functional groups, the graphene nanoribbons possess finite difference in the electrostatic potential between two edges, indicating that the asymmetrically functionalized nanoribbons exhibit polarization between edges. The potential profiles are sensitive to the functional groups attached to the one of two edges. For the nanoribbon with hydroxyl edge, the electrostatic potential at the hydroxylated edge is deeper by $1.9 \mathrm{eV}$ than that at the $\mathrm{H}$ terminated edge. In contrast, for the remaining nanoribbons studied here, the electrostatic potential at the edge with the functional groups is shallower than that at the $\mathrm{H}$ terminated edge. Furthermore, the electrostatic potential difference between the edges depends on the functional groups: The calculated potential differences between edges are 4.31, 2.73, and $1.09 \mathrm{eV}$ for the edges with ketone, carbonyl, and carboxyl groups, respectively. These facts indicate that the polarity of the edges of graphene nanoribbons is tunable by controlling the functional groups attached to the edges. The higher potential at the edge with ketone, carbonyl, and carboxyl groups are ascribed to unsaturated $\mathrm{O}$ atoms in the functional groups, leading to the upward shift of the electrostatic potential of $\mathrm{C}$ 
(a)

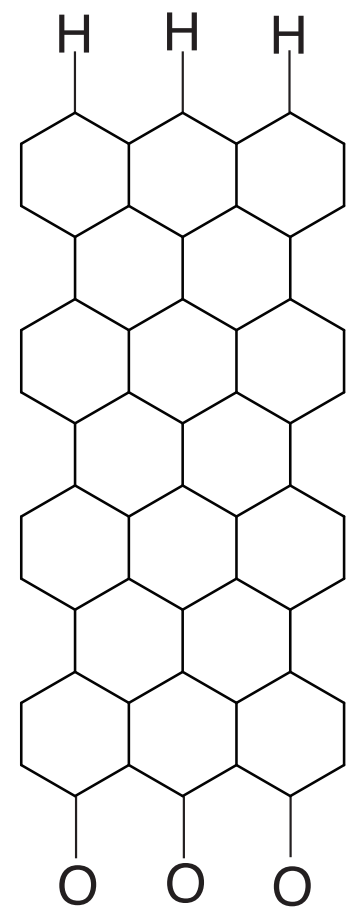

(b)

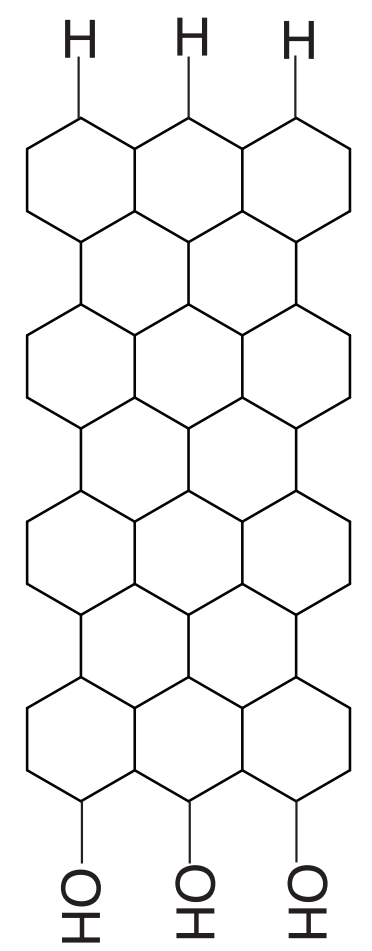

(c)

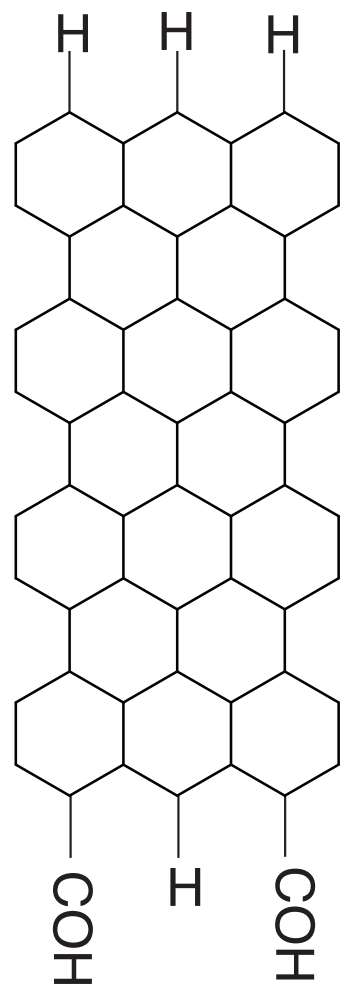

(d)

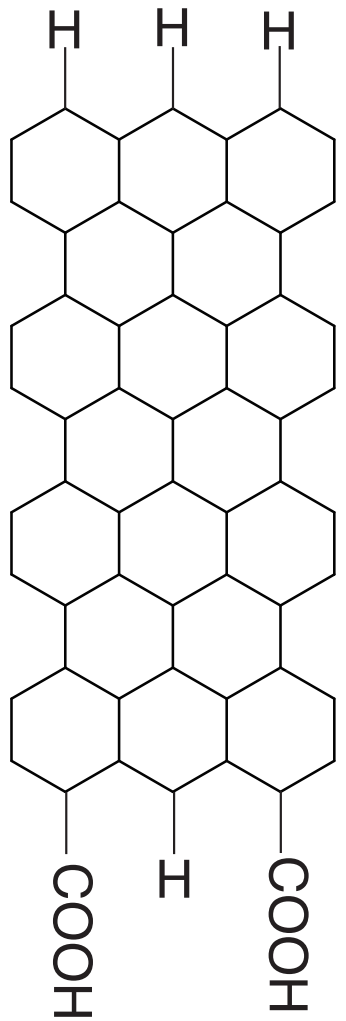

Fig. 1. Structural model of zigzag graphene nanoribbons asymmetrically functionalized by (a) ketone, (b) hydroxyl, (c) carbonyl, and (d) carboxyl groups.

atoms near the edge, causing electron transfer from $\mathrm{C}$ to $\mathrm{O}$ atoms.

In accordance with the polarity of the nanoribbon with functional groups, electronic structure of the nanoribbons exhibits interesting variations. Figure 3 shows the electronic energy band of the graphene nanoribbons one of which edges is functionalized by ketone, hydroxyl, carbonyl, and carboxyl groups. The nanoribbons with ketone and hydroxyl groups exhibit unusual electronic structure near the Fermi level, which are different from the graphene nanoribbons with hydrogenated zigzag edges. The Hterminated nanoribbons with zigzag edges has a pair of flat dispersion bands at the Fermi level and around the zone boundary of one-dimensional Brillouin zone, as known to the edge state. ${ }^{12,13)}$

For the nanoribbons with ketone group [Fig. 3(a)], the nanoribbon is a metal in which two small dispersion bands cross the Fermi level. One of two states exhibits its less dispersion feature around the $\Gamma$ point. For the ribbon with hydroxyl group [Fig. $3(\mathrm{~b})$ ], the nanoribbon possesses two flat dispersion bands in addition to the states with 
parabolic dispersion near the $\Gamma$ point and the Fermi level. These three states are almost degenerated each other at the $\Gamma$ point. In contrast, for the nanoribbons with carbonyl and carboxyl groups [Figs. 3(c) and 3(d)], these nanoribbons exhibits characteristic band structure of the graphene nanoribbon with hydrogenated zigzag edges near the Fermi level. The almost degenerated two states possess flat dispersion around the $\Gamma$ point and at the Fermi level. In addition, a nearly flat dispersion band throughout the Brillouin zone just below the Fermi level indicates that the state is associated with the state distributed on the functional groups, because the functional groups are well separated each other owing to the alternative edge functionalization.

To investigate electronic structure modulation associated with the functional groups, we depict the isosurfaces of the squared wave function of the highest branch of the valence band ( $\mathrm{HO})$ and the lowest branch of the conduction band ( $\mathrm{LU}$ ) at the $\Gamma$ point (Fig. 4). For all nanoribbons, the edge-localized state still emerges on the edge $\mathrm{C}$ atoms terminated by $\mathrm{H}$ atom. In contrast, the distribution of the wave function at the functionalized edges is sensitive to the functional groups. For the nanoribbons with carbonyl and carboxyl groups, the LU state is localized at the edge $\mathrm{C}$ atom terminated by $\mathrm{H}$ atom and extended along the edge. The HO state is distributed on both edge $\mathrm{C}$ atom and the functional groups with $\pi$ state nature. Thus, the carbonyl and carboxyl groups do not affect the edge state. Note that the flat band just below the edge states has a character of the $\sigma$ state of functional groups.

In the case of the nanoribbon with ketone group, the HO state is perfectly localized on the edge $\mathrm{C}$ atoms terminated by $\mathrm{H}$ atom and extended along the ribbon direction, indicating its edge states nature. In contrast, the LU state does not exhibit edge state nature. The state is primary distributed on the $\mathrm{O}$ atoms with $\mathrm{p}_{x}$ nature and the bonding $\sigma$ states between $\mathrm{C}$ and $\mathrm{O}$ atoms.

For the nanoribbon with the hydroxyl group, we find an unusual electronic state at the Fermi level. Two of three states are distributed at the edge $\mathrm{C}$ atomic sites, exhibiting edge state nature. The $\mathrm{HO}$ and $\mathrm{LU}+1$ states have the same distribution to that of the usual edge states of graphene nanoribbon with hydrogenated zigzag edges. However, the remaining state (the LU state) possesses quadratic dispersion and exhibits unusual distribution. The maximum of the state is distributed outside the hydroxylated edges with approximately $3 \AA$ vacuum region. Furthermore, the state is extended in the direction parallel to the ribbon with almost uniform distribution in the vacuum region. The characteristic distribution of the state as well as its quadratic dispersion allows it to be 
classified as the NFE state at the edge of the two dimensional materials. The NFE states usually emerge a few eV above the Fermi level in the surfaces and edges of graphene and h-BN. ${ }^{28-34)}$ The hydroxylation of the zigzag edges of graphene nanoribbons causes the substantial downward shift of the NFE state and injects electrons into the state. Thus, the hydroxylation of the graphene edges may cause the negative electron affinity at the edge, allowing the functionalized graphene nanoribbons constituent materials for field emission sources.

In summary, we studied the geometric and electronic structure of graphene nanoribbons with zigzag edges which are asymmetrically terminated by $\mathrm{H}$ and functional groups $R$ where $R$ are ketone, hydroxyl, carbonyl, and carboxyl groups, using the first-principles total-energy calculations within the framework of the density functional theory. Our calculations show that the work function and electronic structure of graphene edges are sensitive to the functional group species. The work function of the edge functionalized by hydroxyl groups is smaller than that of the hydrogenated edges. In contrast, the work functions of the edge functionalized by ketone, carbonyl, and carboxyl groups are larger than that of the hydrogenated edges. For the edges with the hydroxyl group, we found that the NFE state substantially shifts downward and crosses the Fermi level, indicating the possibility of the negative electron affinity at the hydroxylated zigzag edge of graphene nanoflakes.

\section{Acknowledgements}

One of the authors, AY, acknowledges a Grant-in-Aid for JSPS Fellows. This work was supported by CREST, from the Japan Science and Technology Agency, JSPS KAKENHI Grant Numbers JP25246010, JP16H00898, and JP16H06331 from Japan Society For the Promotion of Science, and the Joint Research Program on Zero-Emission Energy Research, Institute of Advanced Energy, Kyoto University. Part of the calculations was performed on an NEC SX-Ace at the Cybermedia Center at Osaka University and on an SGI ICE XA/UV at the Institute of Solid State Physics, The University of Tokyo. 
(a)

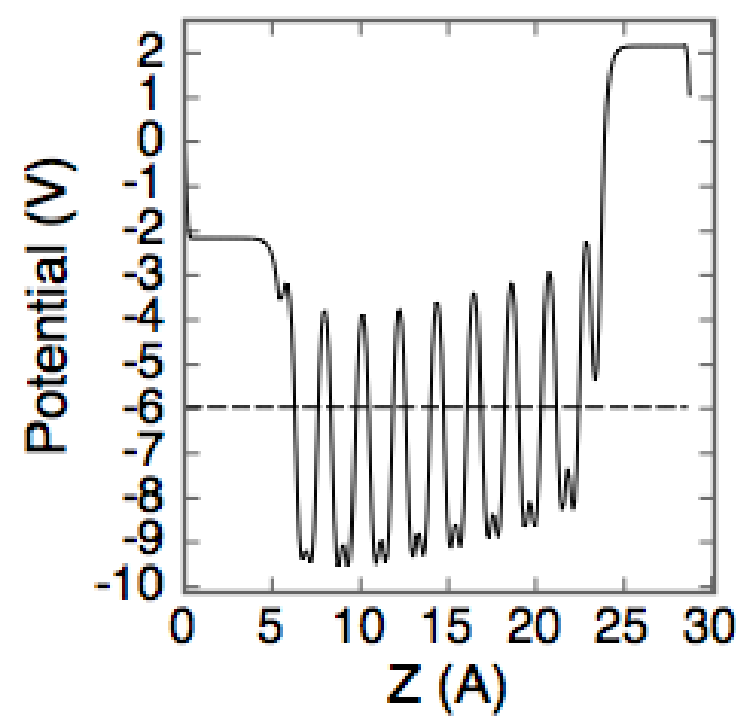

(c)

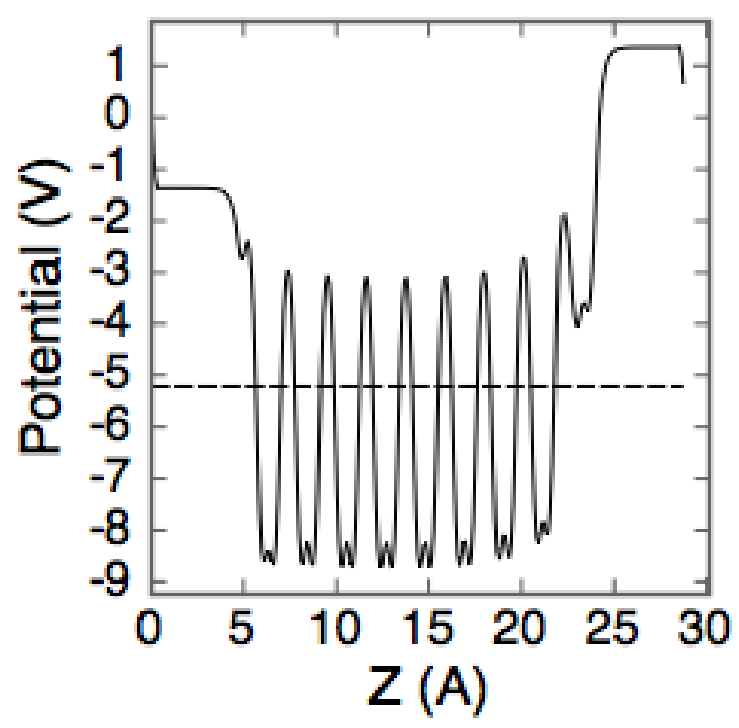

(b)

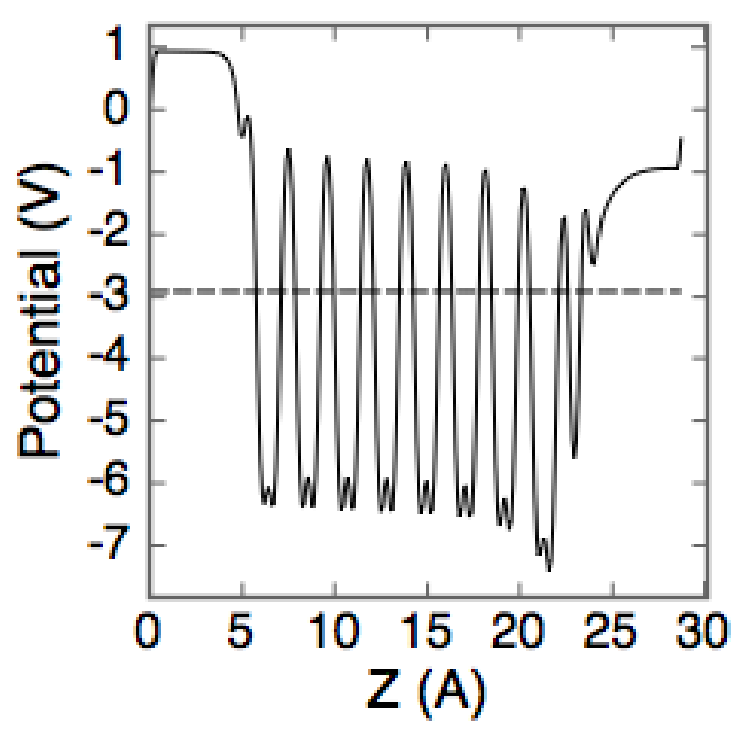

(d)

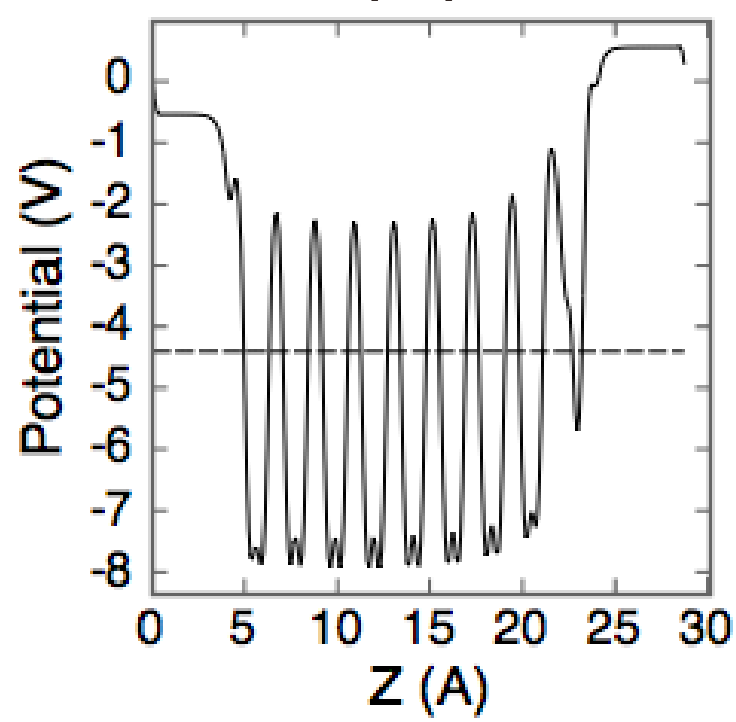

Fig. 2. Plane-averaged electrostatic potential of zigzag graphene nanoribbons asymmetrically functionalized by (a) ketone, (b) hydroxyl, (c) carbonyl, and (d) carboxyl groups. In each figure, the left and right edges are terminated by $\mathrm{H}$ and functional groups, respectively. Horizontal red dotted line denote the Fermi level energy. 

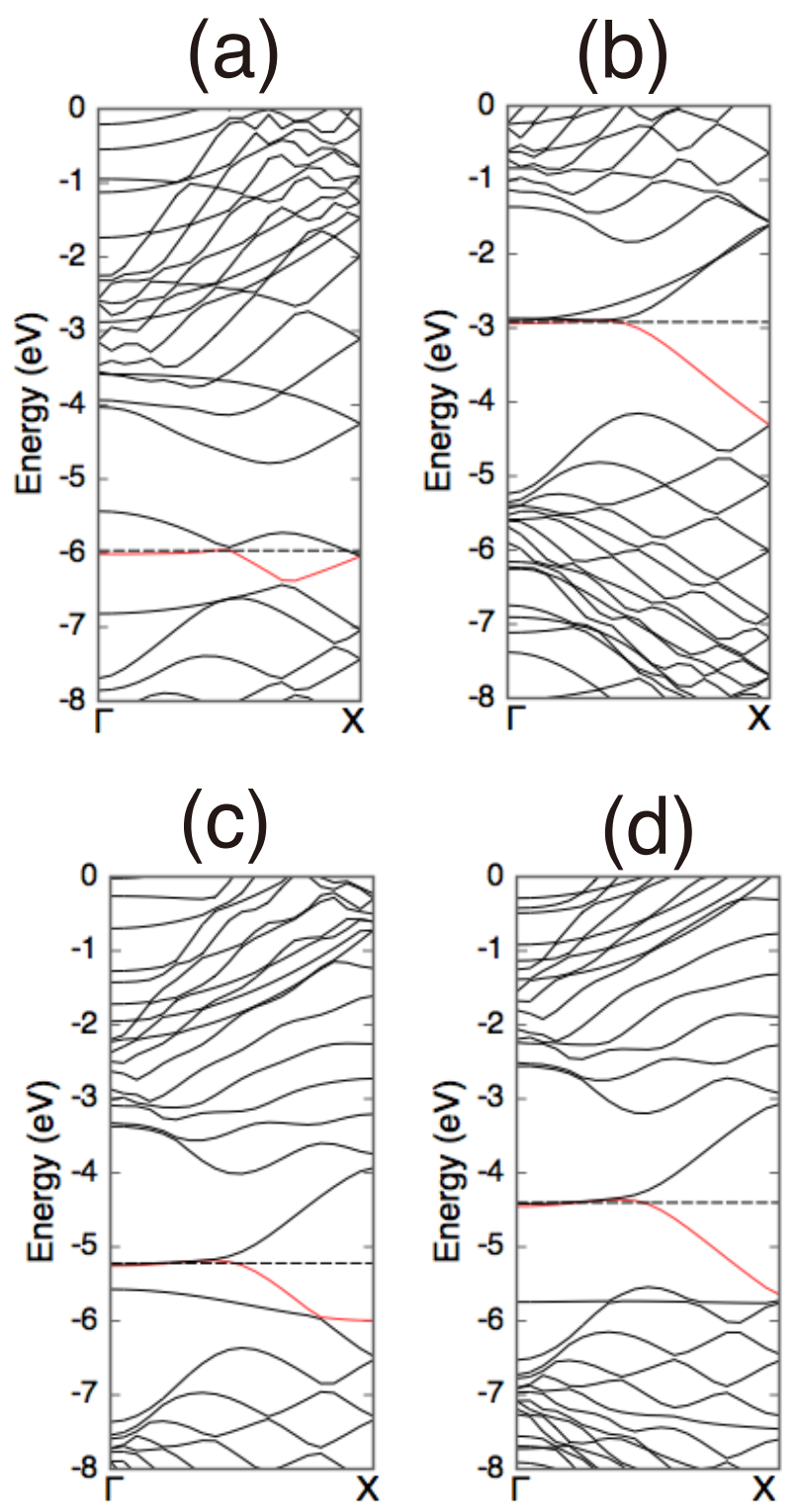

Fig. 3. Electronic energy band of zigzag graphene nanoribbons asymmetrically functionalized by (a) ketone, (b) hydroxyl, (c) carbonyl, and (d) carboxyl groups. Horizontal dotted lines denote the Fermi level energy. The energies are measured from the electrostatic potential energy at the vacuum region outside the $\mathrm{H}$-terminated edge. 
(a)

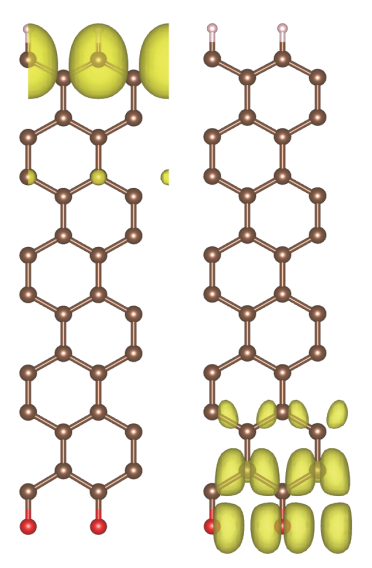

(c)

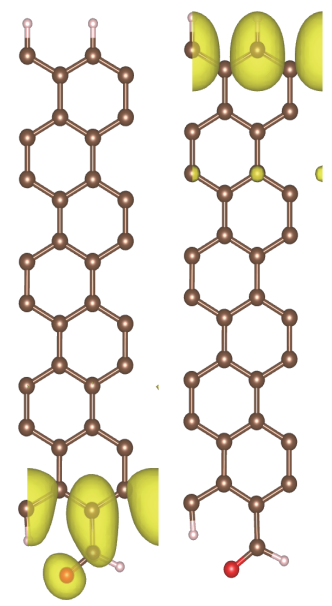

(b)

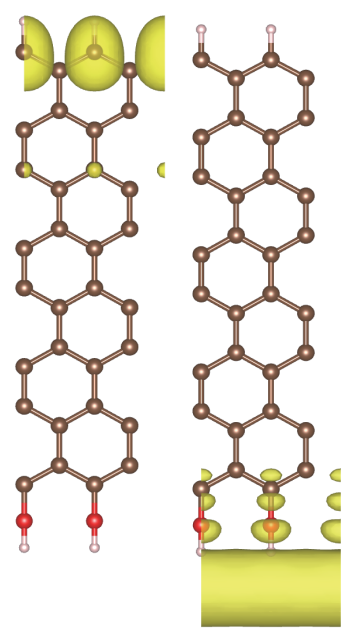

(d)

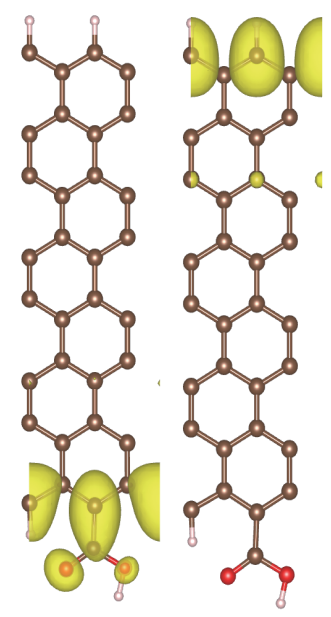

Fig. 4. Isosurfaces of the squared wave function of the top of valences and the bottom of conduction band of zigzag graphene nanoribbons asymmetrically functionalized by (a) ketone, (b) hydroxyl, (c) carbonyl, and (d) carboxyl groups. In each figure, the right and left panels correspond to the $\mathrm{HO}$ and LU states, respectively. Brown, red, and white balls denote $\mathrm{C}, \mathrm{O}$, and $\mathrm{H}$ atoms, respectively. 


\section{References}

1) K.S. Novoselov, A.K. Geim, S.V. Morozov, D. Jiang, Y. Zhang, S.V. Dubonos, I.V. Grigorieva, and A.A. Firsov, Science 306, 666 (2004).

2) K.S. Novoselov, A.K. Geim, S.V. Morozov, D. Jiang, M.I. Katsnelson, I.V. Grigorieva, S.V. Dubonos, and A.A. Firsov, Nature 438, 197 (2005).

3) Y. Zhang, Y.-W. Tan, H.L. Stormer, and P. Kim, Nature 438, 201 (2005).

4) J.B. Oostinga, H.B. Heersche, X. Liu, A.F. Morpurgo, and L.M.K. Vandersypen, Nature Mater. 7151 (2007).

5) Y. Zhang, T.-T. Tang, C. Girit, Z. Hao, M.C. Martin, A. Zettl, M.F. Crommie, Y.R. Shen, and F. Wang, Nature 459, 820 (2009).

6) M.F. Craciun, S. Russo, M. Yamamoto, J.B. Oostinga, A.F. Morpurgo, and S. Tarucha, Nature Nanotech. 4, 383 (2009).

7) T. Ohta, A. Bostwick, T. Seyller, K. Horn, and E. Rotenberg, Science 313, 915 (2006).

8) S. Y. Zhou, G.-H. Gweon, A.V. Fedorov, P.N. First, W.A. de Heer, D.-H. Lee, F. Guinea, A.H.C. Neto, and A. Lanzara, Nature Mater. 6, 770 (2007).

9) A. Mattausch and O. Pankratov, Phys. Rev. Lett. 99, 076802 (2007).

10) N.-T. Cuong, M. Otani, and S. Okada, Phys. Rev. Lett. 106, 106801 (2011).

11) K. Kamiya, N. Umezawa, and S. Okada, Phys. Rev. B 83, 153413 (2011).

12) M. Fujita, K. Wakabayashi, K. Nakada, K. Kusakabe, J. Phys. Soc. Jpn. 65, 1920 (1996)

13) K. Nakada, M. Fujita, G. Dresselhaus, and M.S. Dresselhaus, Phys. Rev. B 54, 17954 (1996).

14) S. Okada, M. Igami, K. Nakada, and A. Oshiyama, Phys. Rev. B 62, 9896 (2000).

15) M. Maruyama and S. Okada, J. Phys. Chem. C 120, 1293 (2016).

16) F. Cervantes-Sodi, G. Csányi, S. Piscanec, and A.C. Ferrari, Phys. Rev. B 77, 165427 (2008).

17) T. Kuila, S. Bose, A.K. Mishra, P. Khanra, N.H. Kim, J.H. Lee, Prog. Mater. Sci. 57, 1061(2012).

18) I.-Y. Jeon, D. Yu, S.-Y. Bae, H.-J. Choi, D.W. Chang, L. Dai, and J.-B. Baek, Chem. Mater. 23, 3987 (2011).

19) K.-S. Kim, I.-Y. Jeon, S.-N. Ahn, Y.-D. Kwon, and J.-B. Baek, J. Mater. Chem. 21, 7337 (2011). 
20) R. Sekiya, Y. Uemura, H. Murakami, and T. Haino, Angew. Chem. 535619 (2014).

21) P. Hohenberg and W. Kohn, Phys. Rev. 136, B864 (1964).

22) W. Kohn and L.J. Sham, Phys. Rev. 140, A1133 (1965).

23) Y. Morikawa, K. Iwata, and K. Terakura, Appl. Surf. Sci. 169-170 11 (2000).

24) J.P. Perdew and A. Zunger, Phys. Rev. B 23, 5048 (1981).

25) D.M. Ceperley and B.J. Alder, Phys. Rev. Lett. 45, 566 (1980).

26) D. Vanderbilt, Phys. Rev. B 41, 7892 (1990).

27) M. Otani and O. Sugino, Phys. Rev. B 73 (2006) 115407.

28) M. Posternak, A. Baldereschi, A.J. Freeman, E. Wimmer, and M. Weinert, Phys. Rev. Lett. 50, 761 (1983).

29) M. Posternak, A. Baldereschi, A.J. Freeman, and E. Wimmer, Phys. Rev. Lett. 52, 863 (1984).

30) A. Catellani, M. Posternak, A. Baldereschi, and A.J. Freeman, Phys. Rev. B 36, 6105 (1987).

31) X. Blase, A. Rubio, S.G. Louie, and M.L. Cohen, Phys. Rev. B 51, 6868 (1995).

32) Q. Liu, Z. Li, and J. Yang, Chinese J. Chem. Phys. 24, 22 (2011).

33) A. Yamanaka and S. Okada, Appl. Phys. Express 7, 125103 (2014).

34) A. Yamanaka and S. Okada, Sci. Rep. 6, 30653 (2016). 